\title{
Morphological classification of post-AGB stars
}

\author{
Arturo Manchado ${ }^{1,2,3}$, D. Anibal García-Hernández ${ }^{1,2}$, Eva Villaver ${ }^{4}$, \\ and Jean Guironnet de Massas ${ }^{1,5}$ \\ ${ }^{1}$ Instituto de Astrofísica de Canarias, \\ Vía Láctea s/n, La Laguna, E-38205, Spain \\ ${ }^{2}$ Departamento de Astrofísica, Universidad de La Laguna, \\ La Laguna, E-38205, Spain \\ ${ }^{3}$ Consejo Superior de Investigaciones Cient Rficas, E-28006 Madrid, Spain \\ ${ }^{4}$ Departamento de Física Teórica C-XI, Univ. Autónoma de Madrid, E-28049 Madrid, Spain \\ ${ }^{5}$ University Joseph Fourier, Grenoble University Joseph Fourier, 3840 Grenoble, France
}

\begin{abstract}
We present a complete study of the morphology of post-Asymptotic Giant Branch (post-AGB) stars. The post-AGB stage is a very short evolutionary phase between the end of the AGB and the beginning of the Planetary Nebula (PN) stage (between 100 and 10,000 yrs). Post-AGB stars do not show variability and are not hot enough to fully ionize the hydrogen envelope. We have defined the end of the post-AGB phase and the beginning of the PN phase when the star has a temperature of $30000 \mathrm{~K}$. Post-AGB stars have a circumstellar shell that is illuminated by the central stars or partially ionized. However, this circumstellar shell is too small to be resolved by ground-based observations. Thus, we have used the Hubble Space Telescope (HST) database to resolve these shells. 117 post-AGBs were found in this database. Here we present the preliminary results on their morphological classification and the correlation with the galactic latitude. Our preliminary results show that $38 \%$ of the sample are stellar-like (S), 31 $\%$ bipolar (B), $12 \%$ multipolar (M) and $19 \%$ elliptical (E).
\end{abstract}

Keywords. Stars: AGB and post-AGB, stars: winds, outflows, circumstellar matter

\section{Introduction}

An Asymptotic Giant Branch (AGB) star can lose up to $80 \%$ of its mass during its last thermal pulses (Villaver, García-Segura \& Manchado 2002). As has been shown over the past 30 years, the study of the Planetary Nebulae (PNe) morphologies provides valuable information about the late stages of stellar evolution. The processes that lead to the different morphologies observed in PNe (Manchado 2003) are not known in detail.

Several models have been proposed to explain the morphologies observed in evolved PNe: The interaction of stellar winds (e.g. Kwok, S., Purton \& Fitzgerald 1978; Frank et al. 1993; Mellema 1995), rotation (e.g. Calvet \& Peimbert 1983); binaries, common envelope phase, substellar interaction, non-radial pulsations (e.g. Soker 1997; Bond \& Livio 1990; Soker \& Harpar 1992) and magnetic fields (e.g., García-Segura et al. 1999).

However most of AGB stars look spherically symmetrical, (e.g. Mauron \& Huggins 2006). Therefore the study of the post-AGB morphology is very important, because it is the early phase for departure from symmetry.

Because of the short-lived ( $\left.10^{2}-10^{4} \mathrm{yrs}\right)$ nature of the post-AGB transition phase, the number of known objects is scarce. In addition, the physical size of the observed nebulosities is very small (between 1 to 10 arcseconds on the sky), making ground-based observations very difficult. The post-AGB nebulosities can display an incipient bipolar 
structure at a very early stage in the post-AGB phase, as has been demonstrated by the Hubble Space Telescope (HST) observations of different samples of post-AGB stars (e.g., Ueta, Meixner \& Bobrowsky 2000, Sahai et al. 2007, Siódmiak et al. 2008). However, there is a general perception in the literature that most of the observed post-AGB stars display asymmetrical (e.g., bipolar, multi-polar, etc.) structures. This is in an apparent contradiction with the morphologies observed in more complete samples of evolved PNe, where round (25\%), elliptical (58\%) and bipolar and multi-polar (17\%) PNe are observed (Manchado 2003). This contradiction is most likely related to a strong bias when selecting post-AGB samples. Here we present a complete and less biased study of the morphology of post-AGB stars in order to shed some light on the possible reasons of this apparent contradiction between the morphologies observed in PNe and their immediate precursors, the post-AGB stars.

\section{Sample selection}

We have selected a large sample of post-AGB candidates showing infrared colors similar to PNe (Manchado et al. 1989, García-Lario et al. 1997). This sample was complemented with the Torun Catalogue of post-AGB stars and related objects (Szczerba et al. 2007) as well as with previous surveys based on ground-based optical spectroscopy (Suárez et al. 2006) and HST imaging of post-AGB stars (e.g., Ueta et al. 2000 , Sahai et al. 2007, Siódmiak et al. 2008). Note that we have defined the end of the post-AGB phase and the beginning of the PN phase when the star is hot enough (spectral type earlier than B) to fully ionize the hydrogen envelope.

We have searched the HST database (http://archive.stsci.edu/) and downloaded images for 117 post-AGB stars obtained with the ACS, WFPC, and WFPC2 instruments onboard HST. The available images were analyzed and all post-AGB stars were morphologically classified as: stellar-like, round, elliptical, bipolar and multi-polar. The sample of 117 post-AGB stars could be divided in four morphological classes, with $44(38 \%)$ objects being stellar like (S), 37 (31\%) Bipolar (B), 14 (12\%) multi-polar (M) and 22 $(19 \%)$ elliptical (E). It is especially remarkable that non-round post-AGB were found (although, as we will see later, there is an explanation), to the contrary of what is found in $\mathrm{PNe}(25 \%)$.

\section{Results}

One of the most striking results is the lack of round post-AGB, contrary to what was found by Manchado (2003). However, five post-AGBs that were classified as ellipticals show well defined bow-shocks with the interstellar medium (ISM).

Villaver, Manchado, \& García-Segura (2012), have shown that the interaction with the ISM is a powerful mechanism in the creation of asymmetric structures during the AGB phase. With velocities of the interaction as low as $10 \mathrm{~km} \mathrm{~s}^{-1}$ and over a range of ISM density conditions, they were able to create bow-shock structures that, with a few exceptions, will survive into the later stages of the evolution. The post-AGB stars affected by this mechanism will be observed as non-spherical, and the source of their non-spherical nature is the interaction of the ejected mass-loss with the ISM, and not asymmetrical wind ejections. We propose that a likely mechanism to explain the lack of round post-AGB stars is the interaction with the ISM.

Figure 2 shows the galactic latitude distribution of the different morphologies. We found medium values for $|b|$ of $4.2,2.8,4.9$, and 1.6 for $\mathrm{E}, \mathrm{B}, \mathrm{M}$ and $\mathrm{S}$ types. These values compare well with the PNe galactic distribution Manchado (2003) $(|b|=7.8,4.9$, 2.3 and 2.1 for types R, E, B and M respectively), especially for the E and B type. It is 

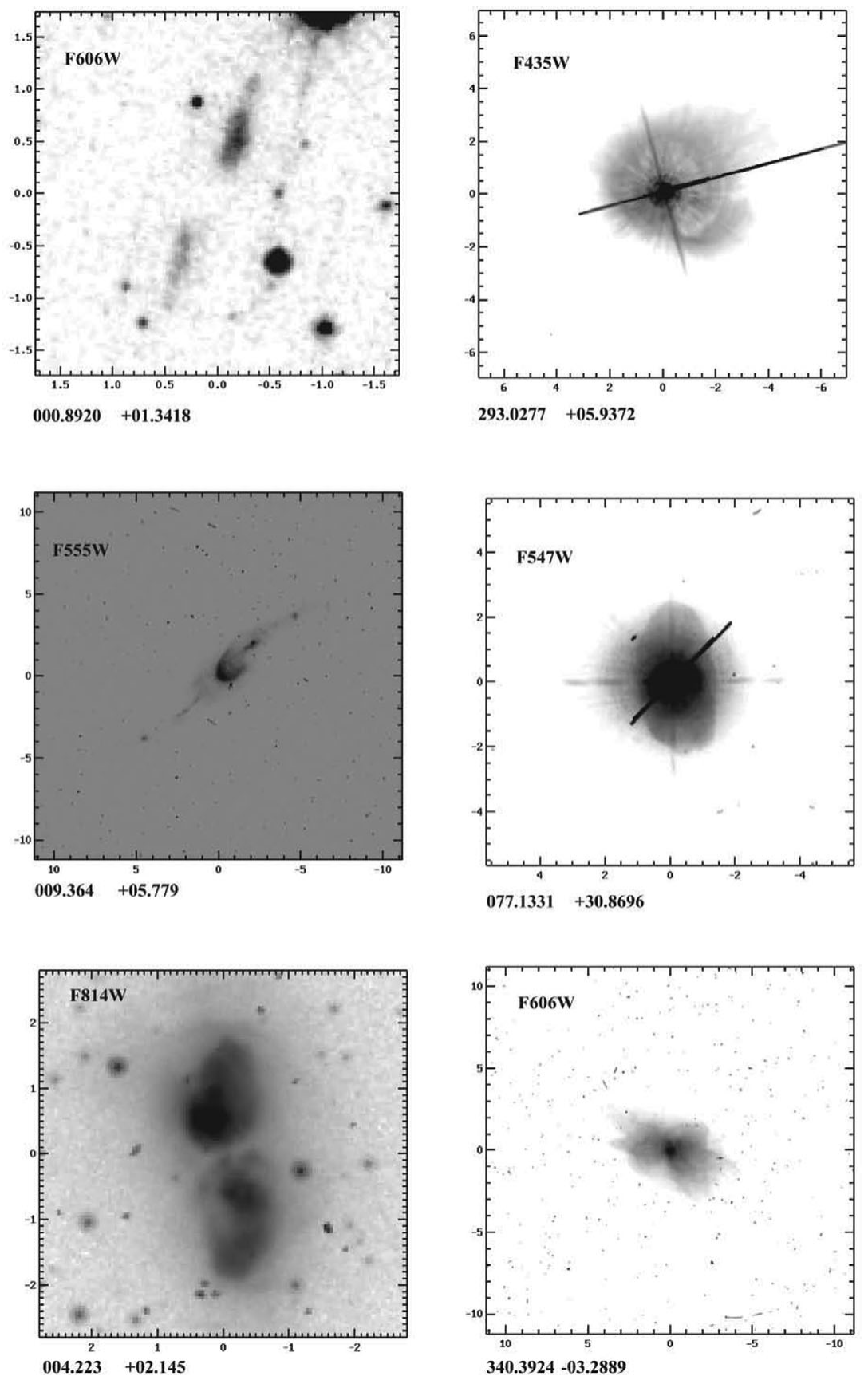

$340.3924-03.2889$

Figure 1. Example of each of the different morphologies observed in post-AGB stars: bipolar (left column), elliptical (upper and middle right column) and multi-polar (bottom right column). The scale is in arcseconds, North is up and East to the left.

noteworthy that the S-types have the lowest galactic latitude. However, they also show a higher concentration near the Galactic Bulge, which may imply that they are further away and thus the HST was not able to resolve them. Alternatively, their precursor stars may be the most massive ones. 


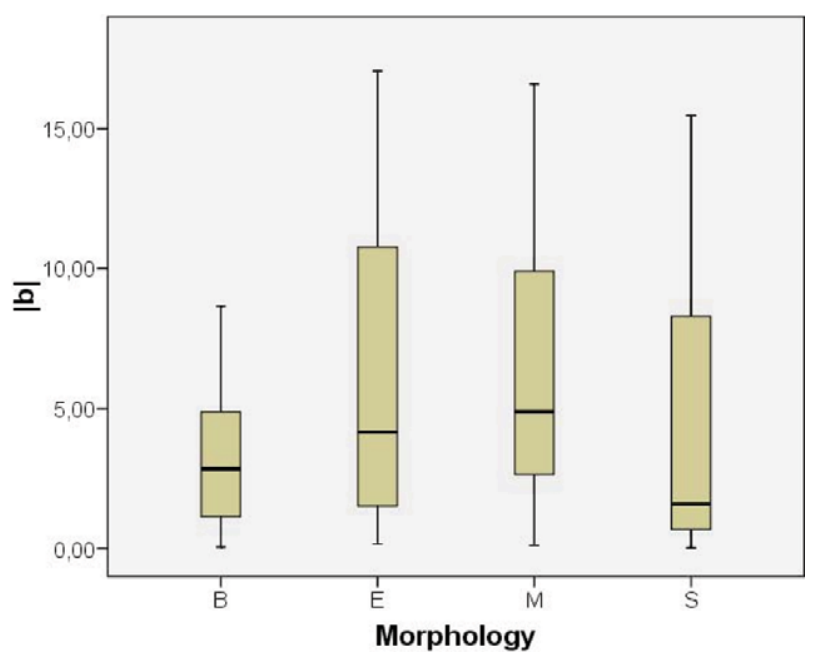

Figure 2. Galactic distribution, in quartiles, for the post-AGB morphological classes.

The different galactic distribution (in quartiles) for the E, B and $\mathrm{M}$ types may imply that each morphological type came from a different star population.

The different galactic distribution among the $\mathrm{B}$ and $\mathrm{M}$ types is consistent with the model proposed by García-Segura \& Lopez (2000) where the most massive stars will form B types, and binary systems will form the M type.

\section{Acknowledgments}

D.A.G.H. and A.M. acknowledge support for this work provided by the Spanish Ministry of Science and Innovation (MICINN) under a JdC grant and under grant AYA2007-64748. EV acknoledges suport from grant AYA2010-20630.

\section{References}

Bond, H. E. \& Livio, M. 1990, ApJ, 355, 568

Calvet, N. \& Peimbert, M., 1983, RMxAA, 5, 319

García-Lario, P., Manchado, A., Pych, W., \& Pottasch, S. R. 1997, A\&AS, 126, 479

García-Segura, G., Langer, N., Różyczka, M., \& Franco, J. 1999, ApJ, 517, 767

García-Segura, G. \& Lopez, J. A. 2000, ApJ, 544, 336

Kwok, S., Purton, C. R., \& Fitzgerald, P. M. 1978, ApJ, 219, L125

Manchado, A., García-Lario, P., Esteban, C., Mampaso, A., \& Pottasch, S. R. 1989, A\&SAS, 214,139

Manchado, A. 2003, in Planetary Nebulae: Their Evolution and Role in the Universe, IAU Symp. 209 eds. S. Kwok, M. Dopita, \& R. Sutherland. PASP, p., 431

Mauron, N. \& Huggins, P. J. 2006, A\& A, 452, 257

Sahai, R., Morris, M., Sánchez Contreras, C., \& Claussen, M. 2007, AJ, 134, 2200

Siódmiak, N., Meixner, M., Ueta, T., et al. 2008, ApJ, 677, 382

\&Soker, N., 1997, ApJS, 112, 487S

Soker, N. \& Harpar, A. 1992, PASP, 104, 923

Suárez, O., García-Lario, P., Manchado, A., et al. 2006, A $₫ A, 458,173$

Szczerba, R., Siódmiak, N., Stasińska, G., \& Borkowski, J. 2007, A\&A, 469, 799

Ueta, T., Meixner, M., \& Bobrowsky, M. 2000, ApJ 528, 861

Villaver, García-Segura, G. \& Manchado, A. 2002, ApJ, 571, 880

Villaver, E., Manchado, A., \& García-Segura, G. 2012, ApJ, 748, 94 